\title{
Delayed-onset of progressive pseudorheumatoid dysplasia in a Chinese adult with a novel compound WISP3 mutation: a case report
}

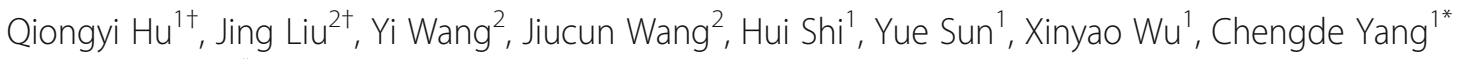
and Jialin Teng ${ }^{1 *}$

\begin{abstract}
Background: Progressive pseudorheumatoid dysplasia (PPD) is a rare autosomal recessive genetic disease that is characterized by pain, stiffness and enlargement of multiple joints with an age of onset between 3 and 8 years old. Mutations in the WISP3 (Wnt1-inducible signal pathway) gene are known to be the cause of PPD.

Case presentation: We present a case of delayed-onset PPD in a Chinese man. The 35-year-old proband presented with an almost 20-year history of pain and limitations in mobility in multiple joints. Based on the clinical manifestations, the patient was diagnosed with PPD; however, there was no specific evidence to confirm this diagnosis. Through mutational analyses, two WIPS3 mutations in exon 4, including a novel frameshift mutation (c.670dupA) in the paternal allele and an already described nonsense mutation (c.756C > A, p.Cys $252^{*}$ ) in the maternal allele, were identified in the proband. Thus, the patient was diagnosed with PPD. Furthermore, we found that the proband's son only carried one of the mutations (c.670dupA) and therefore determined that he would not be affected by PPD in the future.

Conclusions: In this case, we successfully diagnosed the disease that the proband was affected precisely after the reunion of clinical diagnosis and genetic analysis. These findings demonstrate the clinical utility of genetic analysis to diagnose skeletal dysplasia and guide genetic counseling.
\end{abstract}

Keywords: Progressive pseudorheumatoid dysplasia-PPD-WISP3-skeletal dysplasia-mutation

\section{Background}

Progressive pseudorheumatoid dysplasia (PPD; MIM208230) is an extremely rare and inheritable autosomal recessive disease with an approximate incidence of one per million in the UK [1]. This disorder is characterized by noninflammatory arthropathy. The signs and symptoms of the disorder typically begin between the ages of 3 and 8 , and the typical manifestations include pain, stiffness and limitation in motion of multiple joints in the absence of extra-skeletal involvement and bone erosion [2]. Patients with atypical presentations manifest as seronegative arthropathy either under 3 years old or above 8 years old [3].

\footnotetext{
* Correspondence: yangchengde@sina.com; tengteng8151@sina.com ${ }^{\dagger}$ Equal contributors

${ }^{1}$ Department of Rheumatology and Immunology, Ruijin Hospital, Shanghai Jiao Tong University School of Medicine, Shanghai 200025, China

Full list of author information is available at the end of the article
}

PPD has been attributed to the homozygous or compound heterozygous loss-of-function mutations in WISP3 (Wnt1-inducible signal pathway; MIM: 603,400), which is located on chromosome 6q22 [4]. WISP3 is a member of the connective tissue growth factor $(\mathrm{CCN})$ family of cysteine-rich growth factors [5]. It has been reported that WISP3 influences cartilage homeostasis and bone growth [4]. To date, more than 64 different WISP3 mutations have been reported in more than 200 PPD individuals [6], with only 18 from Chinese patients.

Here, we report a case of delayed-onset PPD in a 35-year old Chinese man. Using genetic analysis, we identified a novel compound WISP3 mutation in exon 4, a frameshift mutation (c.670dupA) in the paternal allele and a nonsense mutation (c.756C > A, p.Cys $252^{*}$ ) in the maternal allele in 
the proband. Additionally, we found that the proband's son only carried one mutation (c.670dupA).

\section{Case presentation}

The proband, a 35-year old man, was referred to us for a 20-year history of pain and limitation in mobility of multiple joints and a one-year history of worsening symptoms. He developed kyphosis at the age of 11 . The patient endorsed pain in his palms, wrists and elbow joints without the presence of a rash or erythematous swelling. Subsequently, he progressed to develop pain and restricted movements in his hip and ankle joints that was associated with limited motion with squatting. A suspected diagnosis of congenital spondyloepiphyseal dysplasia was made at another hospital, and the doctor recommended he follow up without any therapy. The patient noted pain in his hips and knee joints, and he had difficulty in standing and sitting. The patient developed claudication and was only treated with occasional painkillers instead of systemic therapy.

At the age of 34, the patient was unable to walk without a crutch due to the pain and severely restricted motion in his hip joints after becoming very tired. At a local hospital, a laboratory test showed a normal erythrocyte sedimentation rate $(2 \mathrm{~mm} / \mathrm{h})$, and tests for HLA-B27 remained negative. A radiograph of his hips showed sacroiliitis. The patient was suspected to have ankylosing spondylitis, and he was referred to our institution for further evaluation.
Examination of the spine showed an abnormal spinal curvature with limited range of motion in the cervical spine. Flexion contractures of the elbows and knees were observed, and there was limited range of motion in the elbows, shoulders, knees and ankles. The Patrick test and occiput-to-wall distance test were positive. The rest of his physical examination was unremarkable.

Radiological findings revealed the following: a reduced interarticular space of the hip joints with bilateral femoral neck aseptic necrosis (Fig. 1a); enlargement of the interphalangeal joints (Fig. 1b); osteosclerosis and irregular contour of the joint surface and narrowing of the spaces in the knee joints (Fig. 1c); herniated disks in the thoracolumbar spine with lumbar spine hyperplasia (Fig. 1d) and platyspondyly of the cervical spine (Fig. 1e).

The rest of his five family members including his parents, wife and son did not have any evidence of skeletal dysplasia. Subsequently, the diagnosis of ankylosing spondylitis was challenged, and PPD was strongly suspected. A genetic analysis was carried out, following the CARE guidlines. We found that the proband carried a frameshift mutation (c.670dupA) and a nonsense mutation (c.756C > A, p.Cys252*) in exon 4 of WISP3 (Fig. 2a, b), which have been reported previously $[7,8]$. Furthermore, the frameshift mutation (c.670dupA) was found in the paternal allele (Fig. 2c), and the nonsense mutation (c.756C $>\mathrm{A}$, p.Cys252*) was found in the maternal allele (Fig. 2d). The a

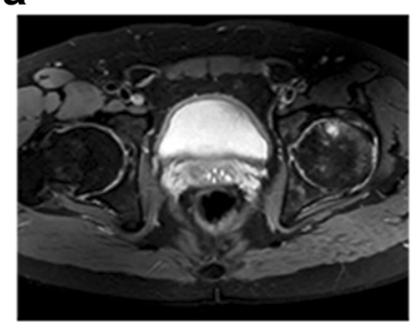

C

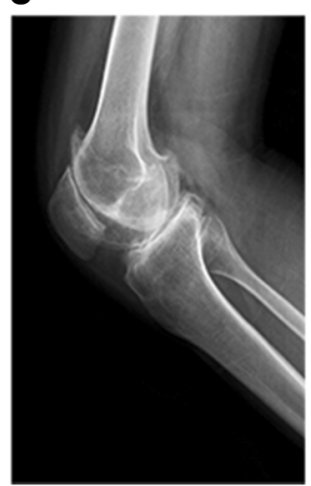

d

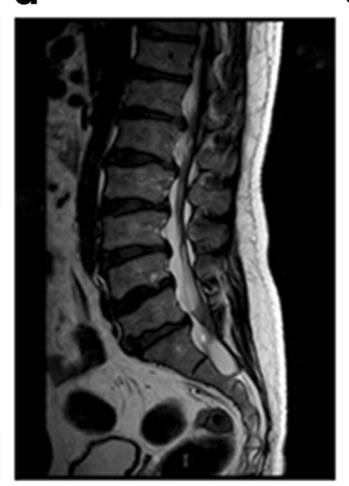

b

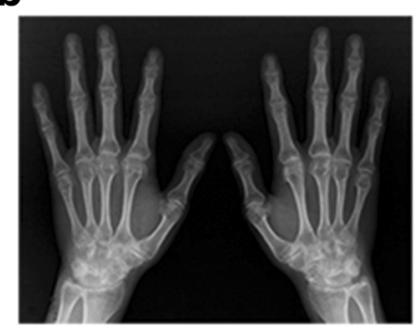

e

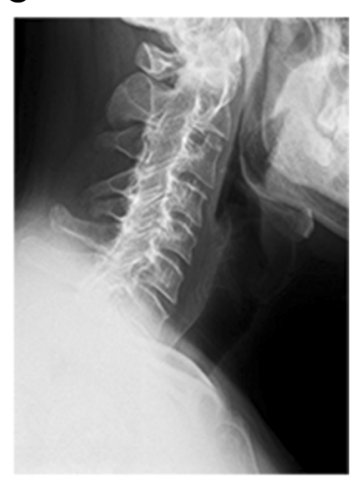

Fig. 1 Radiographs of the proband at the age of 35 years. a Reduced interarticular spaces in the hip joints with bilateral femoral neck aseptic necrosis, $\mathbf{b}$ enlargement of interphalangeal joints, c narrowing of elbow joint space, $\mathbf{d}$ osteosclerosis and an irregular contour of the joint surface and narrow spaces in the knee joints, e herniated disks in the thoracolumbar spine (T12/L1-L5/S1) with lumbar spine hyperplasia, (f) and flattened vertebral bodies in the cervical spine can be noted 
a
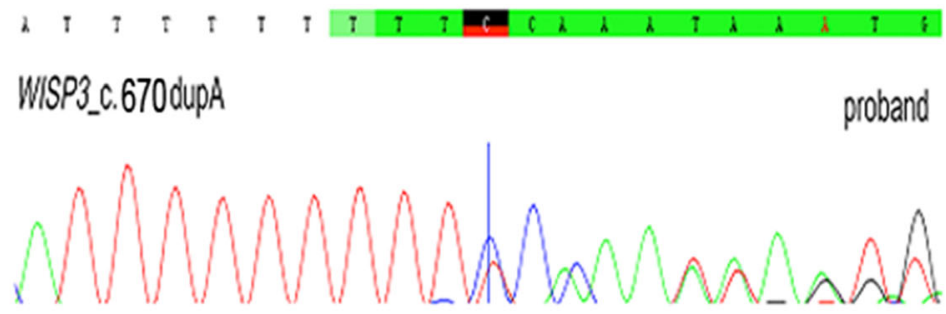

b
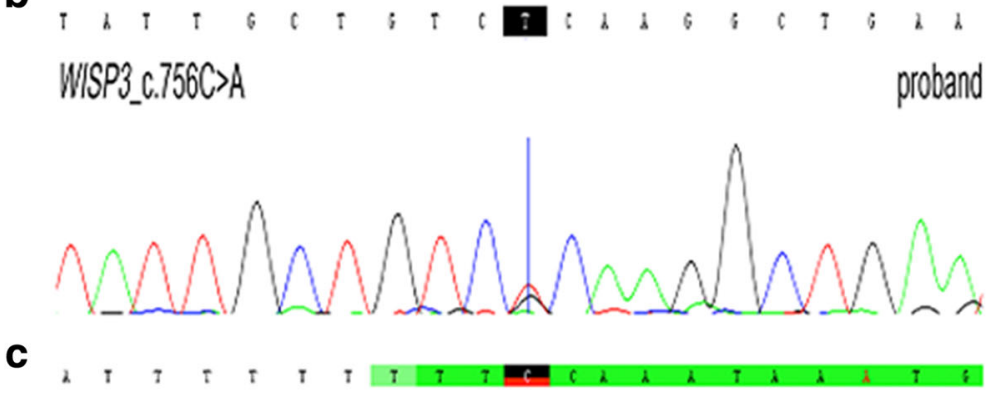

WISP3_c. 670dupA

father of the proband

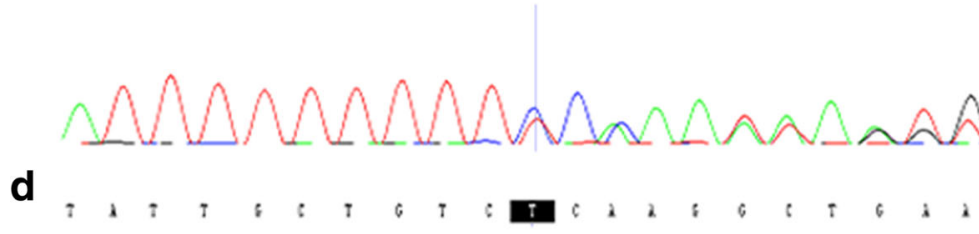

WISP3C. $756 C>A$

mother of the proband

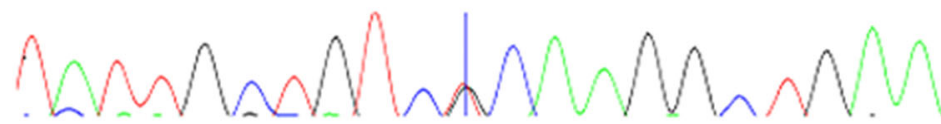

e
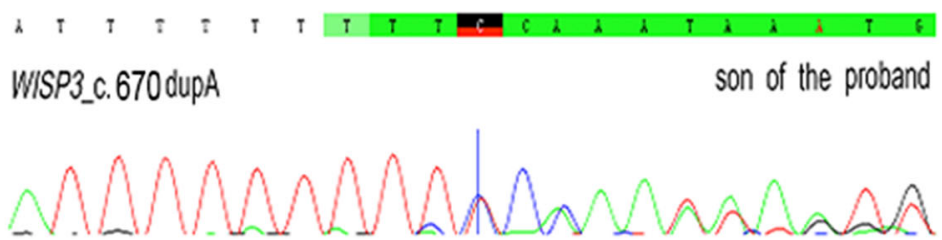

Fig. 2 Sanger sequencing of WISP3 mutations in the five family members. a, b Compound heterozygous mutations, including c.670dupA and c.756C > A, were found in the proband. c The c.670dupA mutation was inherited from the father. $\mathbf{d}$ The c.756C > A mutation was inherited from the mother. e The son carried the c.670dupA mutation

son of the proband was found to carry the frameshift mutation (c.670dupA) inherited from his grandfather (Fig. 2e).

A diagnosis of PPD was confirmed in the proband after combining the clinical characteristics and the results from the genetic analysis. Furthermore, genetic counseling was given for the son, who was told that he would not be affected by PPD in the future.

\section{Discussion and conclusions}

PPD is a rare and inheritable skeletal dysplasia. In this report, we describe a 35-year old proband who suffered from non-inflammatory pain, stiffness and limitation in his mobility of multiple joints for over 20 years without a definite diagnosis. The proband had a normal blood laboratory test. Radiographs showed a narrowing of multiple joint spaces, enlargement of interphalangeal joints, herniated intervertebral disks, and platyspondyly.

Unlike other congenital skeletal dysplasias, patients with PPD are almost completely asymptomatic in early childhood [9]. The resemblance to juvenile idiopathic arthritis and lack of inflammatory biomarkers are the common causes of misdiagnosis. These diagnoses can be 
excluded by laboratory findings and genetic counseling [10]. Moreover, as patients younger than 3 years old or older than 8 years old can have atypical presentations of PPD [3], a diagnosis of PPD was initially considered. Genetic analysis was subsequently performed to confirm our original suspected diagnosis of PPD. To date, the incidence of PPD in China has not been described, and there is an urgent need to determine the spectrum of mutations in Chinese PPD patients and the relationship between the phenotype and genotype of PPD.

The causative role of WISP3 mutations in PPD was first reported in 1999 [4]. Subsequently, a total of 18 WISP3 mutations located in exons 2 to 5 have been described in China (Table 1) $[2,7,8,11-15]$. They include five different mutations (two frameshift mutations and one nonsense mutation) located in exons 2 and 3 (two missense mutations), respectively, as well as five mutations in exon 5 (three frameshift mutations, one nonsense mutation and one missense mutation). To date, 8 WISP3 gene mutations in exon 4 (c. 624delA, c.624_625insA, c.667 T> G, c.679dupA, c.716_722delAAATGAG, c.721 T > G, c.727_733delGAGAAAA, c.756C > A) have been identified in the Chinese population $[7,8,13,14]$.

In our study, we found compound heterozygous mutations (c.670dupA/ c.756C > A) in exon 4 of WISP3 in an atypical PPD patient. These findings are different from the compound mutations in other Chinese patients with PPD previously reported by Ye et al. and Luo et al. [7, 8], indicating the genetic heterogeneity of PPD. Furthermore, to

Table 1 Summary of all currently known WISP3 gene mutations in PPD patients in China

\begin{tabular}{lllll}
\hline No. & Location & Nucleotide change & Amino acid change & References \\
\hline 1 & Exon 2 & c.105dupT & p.Gly36fs*10 & {$[14]$} \\
2 & Exon 2 & c.208_209insA & $/$ & {$[8]$} \\
3 & Exon 2 & c.136C > T & p.Gln46* & {$[2,8,13]$} \\
4 & Exon 3 & c.342 T > G & p.Cys114Trp & {$[8,12-15]$} \\
5 & Exon 3 & c.342G > A & p.Cys114Try & {$[2]$} \\
6 & Exon 4 & c.624delA & p.Lys208fs*24 & {$[14]$} \\
7 & Exon 4 & c.624_625insA & p.Cys209fs 229 & {$[8]$} \\
8 & Exon 4 & c.667 T > G & p.Cys223Gly & {$[7,8]$} \\
9 & Exon 4 & c.679dupA & p.Cys227Leufs ${ }^{*} 21$ & {$[15]$} \\
10 & Exon 4 & c.716_722delAAATGAG & p.Glu239fs*16 & {$[12]$} \\
11 & Exon 4 & c.721 T > G & p.Cys241Gly & {$[15]$} \\
12 & Exon 4 & c.729_735delGAGAAAA & p.Glu243fs ${ }^{*} 255$ & {$[8,13]$} \\
13 & Exon 4 & c.756C > A & p.Cys252* & {$[7]$} \\
14 & Exon 5 & c.840delT & p.Phe280Leufs*33 & {$[11]$} \\
15 & Exon 5 & c.866_867insA & p.Gln289fs*31 & {$[8,12]$} \\
16 & Exon 5 & c.866dupA & p.Ser290Glufs*13 & {$[13]$} \\
17 & Exon 5 & c.857C > G & p.Ser286* & {$[13]$} \\
18 & Exon 5 & c.1000 T >C & p.Ser334Pro & {$[11,12]$} \\
\hline & & & &
\end{tabular}

our knowledge, the c.756dupA WISP3 mutation has not been previously reported in the Gene Bank database. In addition, the c.756C $>$ A nonsense mutation was previously reported by Luo et al. in two patients from the same Chinese family [7]. In contrast, the phenotype in the patient in our study was milder than that of the patients described in their study, and the onset was much later. As typical PPD patients have an onset of symptoms between the ages of 3 and 8 , the diagnosis of PPD in this patient was delayed for 20 years. Ye et al. described two delayedonset PPD cases from the same family [8]. The age of onset was 13 and 14, respectively. Patients carrying a heterozygous mutation (c.208_209insA) show a significant difference in the severity of their PPD phenotype such as, coxa vara, genu varum, and loss of the joint space of the knee with osteoporosis. Due to the genetic heterogeneity of PPD, the correlation between the PPD phenotype and genotype requires further study.

WISP3 is a member of the connective tissue growth factor $(\mathrm{CCN})$ family of cysteine-rich proteins that contains four functional modules. Module 3 is a thrombospondin repeated domain (TSP-1) encoded by exon 4 [5]. TSP-1, a 55-residue consensus sequence of the extracellular matrix glycoprotein, has the ability to bind to a wide range of extracellular targets, such as collagen $\mathrm{V}$, fibronectin, sulphated glycoconjugates and several integrins, suggesting a role in adhesion or interactions with the extracellular matrix [16].

The WISP3 protein appears to have multiple effects on cartilage metabolism and homeostasis by regulating the synthesis of chondrocyte collagen type II and aggrecan, which are essential components in articular cartilage [17]. Zhou et al. found that WISP3 was expressed at low levels in articular cartilage chondrocytes in PPD patients [11], a finding that may explain why PPD mainly affects articular cartilage. However, the pathogenic mechanism of the WISP3 gene in PPD remains unclear; thus, a specific therapy is not yet available [3]. Therefore, an early and definite diagnosis of PPD will be necessary to develop further studies.

In summary, a new combination of two WISP3 mutations in exon 4 was identified in a Chinese adult with delayedonset PPD. Genetic analysis led to a precise diagnosis of PPD. Our study demonstrated the clinical utility of genetic analysis to diagnose skeletal dysplasia and guide genetic counseling.

\footnotetext{
Abbreviations

CCN: Connective tissue growth factor; PPD: Progressive pseudorheumatoid dysplasia; TSP-1: Thrombospondin repeated domain; WIPS: Wnt1-inducible signal pathway

Acknowledgements

We thank for the patient and his family who agree us to use their images and clinical data.
} 


\section{Funding}

This work was supported by the National Natural Science Foundation of China (81373211 and 81671589), the Prairie fire program (LYJH-358), the National Basic Research Program (2014CB541801), and International S\&T Cooperation Program of China (2013DFA30870).

\section{Availability of data and materials}

The data of the current study are available from the corresponding author on reasonable request.

\section{Authors' contributions}

QYH and JL wrote the manuscript. YW and JCW performed the genetic analysis. HS performed the physical examination and radiographic examination. YS and XYW assisted with the data analysis and interpretation. CDY and JLT provided guidance in patient management, participated in the manuscript preparation and revision. All authors have read and approved of the final manuscript.

\section{Ethics approval and consent to participate}

The study was performed in accordance with the Declaration of Helsinki and the principles of Good Clinical Practice. Biological samples were obtained under a protocol approved by the Institutional Research Ethics Committee of Ruijin Hospital (ID: 2016-62), Shanghai, China. The patient and his family signed written informed consent for the report to be published.

\section{Consent for publication}

We confirm that this patient and his family have given their written consents for the case report to be published, including their medical data and images. A copy of the written consent is available for review by the editor of this Journal.

\section{Competing interests}

The authors declare that they have no competing interests.

\section{Publisher's Note}

Springer Nature remains neutral with regard to jurisdictional claims in published maps and institutional affiliations.

\section{Author details}

'Department of Rheumatology and Immunology, Ruijin Hospital, Shanghai Jiao Tong University School of Medicine, Shanghai 200025, China. ${ }^{2}$ State Key Laboratory of Genetic Engineering and Ministry of Education (MOE) Key Laboratory of Contemporary Anthropology, Collaborative Innovation Center for Genetics and Development, School of Life Sciences, Fudan University, Shanghai, China.

Received: 18 August 2017 Accepted: 29 November 2017

Published online: 15 December 2017

\section{References}

1. Wynne-Davies R, Hall C, Ansell BM. Spondylo-epiphysial dysplasia tarda with progressive arthropathy. A "new" disorder of autosomal recessive inheritance. J Bone Joint Surg Br. 1982;64(4):442-5.

2. Yue $H$, Zhang $Z \mathrm{~L}$, He JW. Identification of novel mutations in WISP3 gene in two unrelated Chinese families with progressive pseudorheumatoid dysplasia. Bone. 2009;44(4):547-54.

3. Garcia Segarra N, Mittaz L, Campos-Xavier AB, Bartels CF, Tuysuz B, Alanay Y, Cimaz R, Cormier-Daire V, Di Rocco M, Duba HC, et al. The diagnostic challenge of progressive pseudorheumatoid dysplasia (PPRD): a review of clinical features, radiographic features, and WISP3 mutations in 63 affected individuals. Am J Med Genet C Semin Med Genet. 2012;160C(3):217-29.

4. Hurvitz JR, Suwairi WM, Van Hul W, El-Shanti H, Superti-Furga A, Roudier J, Holderbaum D, Pauli RM, Herd JK, Van Hul EV, et al. Mutations in the CCN gene family member WISP3 cause progressive pseudorheumatoid dysplasia. Nat Genet. 1999;23(1):94-8.

5. Chen CC, Lau LF. Functions and mechanisms of action of CCN matricellular proteins. Int J Biochem Cell Biol. 2009;41(4):771-83.

6. Montane LS, Marin OR, Rivera-Pedroza Cl, Vallespin E, Del Pozo A, Heath KE. Early severe scoliosis in a patient with atypical progressive pseudorheumatoid dysplasia (PPD): identification of two WISP3 mutations, one previously unreported. Am J Med Genet A. 2016;170(6):1595-9.
7. Luo H, Shi C, Mao C, Jiang C, Bao D, Guo J, Du P, Wang Y, Liu Y, Liu X, et al. A novel compound WISP3 mutation in a Chinese family with progressive pseudorheumatoid dysplasia. Gene. 2015;564(1):35-8.

8. Ye J, Zhang HW, Qiu WJ, Han LS, Zhang YF, Gong ZW, Gu XF. Patients with progressive pseudorheumatoid dysplasia: from clinical diagnosis to molecular studies. Mol Med Rep. 2012;5(1):190-5.

9. el- Shanti HE, Omari HZ, Qubain HI. Progressive pseudorheumatoid dysplasia: report of a family and review. J Med Genet. 1997;34(7):559-63.

10. Dalal A, Bhavani GS, Togarrati PP, Bierhals T, Nandineni MR, Danda S, Danda D, Shah H, Vijayan S, Gowrishankar K, et al. Analysis of the WISP3 gene in Indian families with progressive pseudorheumatoid dysplasia. Am J Med Genet A. 2012;158A(11):2820-8.

11. Zhou HD, Bu YH, Peng YQ, Xie H, Wang M, Yuan LQ, Jiang Y, Li D, Wei QY, $\mathrm{He} Y \mathrm{~L}$, et al. Cellular and molecular responses in progressive pseudorheumatoid dysplasia articular cartilage associated with compound heterozygous WISP3 gene mutation. J Mol Med (Berl). 2007;85(9):985-96.

12. Sun J, Xia W, He S, Zhao Z, Nie M, Li M, Jiang Y, Xing X, Wang O, Meng X, et al. Novel and recurrent mutations of WISP3 in two Chinese families with progressive pseudorheumatoid dysplasia. PLoS One. 2012;7(6):e38643.

13. Yu Y, Hu M, Xing X, Li F, Song Y, Luo Y, Ma H. Identification of a mutation in the WISP3 gene in three unrelated families with progressive pseudorheumatoid dysplasia. Mol Med Rep. 2015;12(1):419-25.

14. Liu L, Li N, Zhao Z, Li W, Xia W. Novel WISP3 mutations causing spondyloepiphyseal dysplasia tarda with progressive arthropathy in two unrelated Chinese families. Joint Bone Spine. 2015;82(2):125-8.

15. Yan W, Dai J, Xu Z, Shi D, Chen D, Xu X, Song K, Yao Y, Li L, Ikegawa S, et al. Novel WISP3 mutations causing progressive pseudorheumatoid dysplasia in two Chinese families. Hum Genome Var. 2016;3:16041.

16. Holbourn KP, Acharya KR, Perbal B. The CCN family of proteins: structurefunction relationships. Trends Biochem Sci. 2008;33(10):461-73.

17. Sen M, Cheng YH, Goldring MB, Lotz MK, Carson DA. WISP3-dependent regulation of type $\|$ collagen and aggrecan production in chondrocytes. Arthritis Rheum. 2004:50(2):488-97.

\section{Submit your next manuscript to BioMed Central and we will help you at every step:}

- We accept pre-submission inquiries

- Our selector tool helps you to find the most relevant journal

- We provide round the clock customer support

- Convenient online submission

- Thorough peer review

- Inclusion in PubMed and all major indexing services

- Maximum visibility for your research

Submit your manuscript at www.biomedcentral.com/submit
) Biomed Central 